\title{
Performance Analysis of Underwater Acoustic Communication using IDMA-OFDM-MIMO with Reed Solomon and Turbo Code
}

\author{
Rajashri Khanai ${ }^{1}$ \\ ${ }^{1}$ Department of Electronics and Communication Engineering, KLE's Dr. M.S. Sheshgiri College of Engineering and \\ Technology, Belagavi, India \\ E-mail: rajashri.khanai@gmail.com \\ Salma S. Shahapur ${ }^{2}$ \\ ${ }^{2}$ Jain College of Engineering/Electronics and Communication, Belagavi, 590010, India \\ E-mail: salma.jce@gmail.com \\ Dattaprasad Torse ${ }^{3}$ \\ ${ }^{3}$ Department of Electronics and Communication Engineering, KLS Gogte Institute of Technology, Belagavi, India \\ E-mail: datorse@git.edu
}

Received: 26 September 2018; Accepted: 16 October 2018; Published: 08 December 2018

\begin{abstract}
The underwater acoustic environment is a promising technology which explores the real-time data collection for various applications. However, these channels are prone to errors, and characterized by propagation delay, half duplex communication. At low frequencies, the fading phenomenon extensively affect the behavior of the channel and hence the effect the design of reliable communication system. The underwater acoustic channels to perform appreciably reliable communication, an attempt are made by various modulation and coding techniques. Simulation results for the combination of BPSK modulation with Reed Solomon code (BPSK-RS) having various interleavers Random Interleaver, Matrix Interleaver, have been investigated. To improve the Bit Error Rate performance various modulation techniques such as BPSK, QPSK, and QAM were combined with coding algorithms like RS code, Turbo code and different Interleavers. The investigation of the above combination reveals that IDMA-OFDM-MIMO with BPSK modulation, Turbo code with Random Interleaver technique improves significantly Bit Error Rate performance.
\end{abstract}

Index Terms-IDMA, Interleaver, BPSK, QPSK, QAM.

\section{INTRODUCTION}

Underwater acoustic sensor networks are used in wide range of applications and in commercial. In wireless communication media the underwater acoustic channel is one of the most thought-provoking channels [1], [2]. Underwater acoustic channels existing many challenges for reliable high data rate communications. In underwater communication using acoustic link, the complexity of the channel is created by the ocean environment structures which comprise double side spreading, delay, Doppler spreads, frequency selective fading, absorption at high frequencies, ambient noise at low frequencies and limited bandwidth [3]. The choice of modulation and error detection/correction techniques is very challenging. To protect the transmitted signal against noise and other impairments in underwater acoustic channels, the channel coding is important. Digital communication system of underwater acoustic channels needs to attain high reliability in the occurrence of noise and interference. In this paper, the performance analysis of RS (Reed Solomon) and Turbo channel coding schemes with Random and Matrix interleavers and BPSK, QPSK, QAM modulation techniques are investigated. As the instruments of underwater communication networks are battery functioned so it is important that power consumption must be less. Power consumption is measured by PAPR (Peak to Average Power Ratio).

\section{Acoustic Transmission Loss And Ambient Noise}

For underwater communication, the analytical model for propagation loss and ambient noise is presented. As the sound travels from transmitter to receiver the Acoustic Transmitter Loss (ATL) is the collected decrease in acoustic intensity [4].

\section{A. Absorption Loss and Dispersion Loss}

Acoustic route loss depends on frequency of the signal, distance and absorption [5], [6]. Dispersion loss increases with distance. And also signal experiences a dispersion loss [3]. The complete transmission loss in underwater channel over a distance of meters at a signal frequency $q$ 
is given by

$$
A T L=d . \log l+\operatorname{lol} 10 \log a(q)
$$

$\mathrm{d}$ is dispersion factor (for circular dispersion $\mathrm{d}=2$, cylindrical shaped dispersion $\mathrm{d}=1$, and for practical dispersion $\mathrm{d}=1.5)$ [7]. And $10 \log a(q)$ is the absorption constant it is expressed using Thorp's formula given by

$$
\begin{aligned}
& 10 \log a(f)= \\
& 0.11 \frac{f^{2}}{1+f^{2}}+44 \frac{f^{2}}{4100+f^{2}}+2.75 \cdot 10^{-4} f^{2}+0.003
\end{aligned}
$$

In underwater communication using acoustic link absorption constant increases with signal frequency, and frequency is bounded by it. The dispersion loss rises with frequency as well as with distance [8].

\section{B. Surface Reflection}

The reflection of sound from the ocean surface describes reflection, and surface reflection is affected by the unevenness or evenness of the ocean [9],[10]. Using the Beckmann-Spizzichino surface reflection model the transmission loss on reflection can be calculated when the ocean is uneven, and is given by

$$
R F=10 \log \left(\frac{1+\left(\frac{q}{q_{1}}\right)^{2}}{1+\left(\frac{q}{q_{2}}\right)^{2}}\right)-\left(1+\frac{90-w}{60}\right)\left(\frac{\theta}{30}\right)^{2}
$$

Where $q_{1=\sqrt{10}} q_{2}$ and $q_{2}=378 w^{-2}$

Where the wind speed in knots is $\mathrm{w}$ and $\theta$ is the angle of incidence and its unit is degrees. The total acoustic pathway loss is calculated by using the following equation

$$
P L=d .10 \log l+\operatorname{lo} .10 \log a(q)+R F
$$

\section{Ambient Noise}

Gaussian information and a continuous power spectral density (PSD) describe the ambient noise bases. The following formula gives the power spectral density for waves, shipping turbulence and thermal noise,

$$
\begin{gathered}
10 \log P_{w}(q)= \\
50+7.5 w^{1 / 2}+20 \log q-40 \log (q+0.4) \\
10 \log P_{s}(q)= \\
40+20(s-0.5)+26 \log q-60 \log (q+0.03) \\
10 \log P_{t}(q)=17-(30 \log q) \\
10 \log P_{t h}(q)=-15+20 \log q
\end{gathered}
$$

Where wind speed in $\mathrm{m} / \mathrm{s}$ is represented by $\mathrm{w}$ and shipping activity factor is denoted by s. the overall power spectral density is given by

$$
P(q)=P_{t}(q)+P_{s}(q)+P_{w}(q)+P_{t h}(q)
$$

\section{Underwater SNR}

Underwater communication with acoustic channel, the transmission loss depends on frequency, communication distance and it can be characterised by

$$
P(q)=P_{t}(q)+P_{s}(q)+P_{w}(q)+P_{t h}(q)
$$

\section{IDMA OFDM MIMO}

For reliable data transmission in underwater acoustic communication, IDMA OFDM MIMO (Interleaver Division Multiple Access Orthogonal Frequency Division Multiplexing Multiple Input Multiple Output) technique has been implemented for various coding techniques with different interleavers and different modulation techniques. Performance parameters are compared.

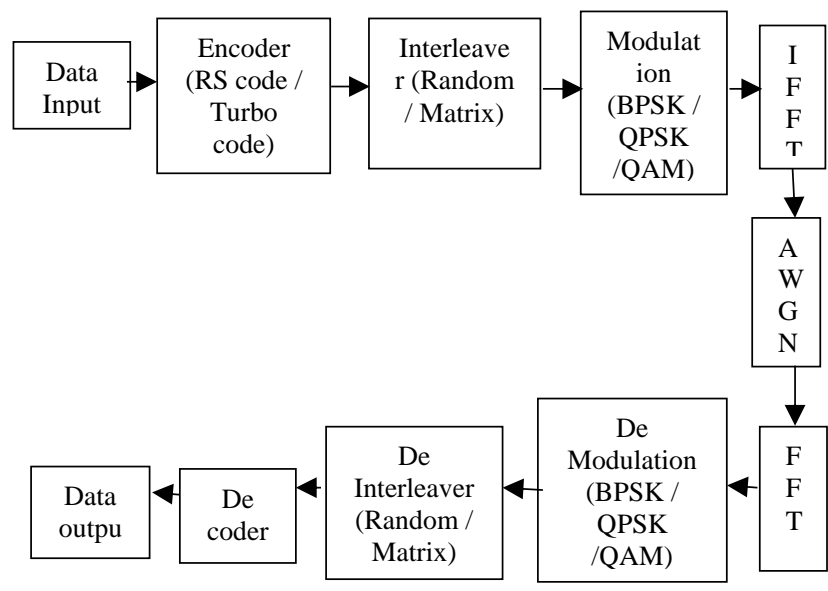

Fig.1. Block diagram of IDMA OFDM MIMO

The input data is encoded with help of RS encoder and interleaving operation is done through Random interleaver. Modulation is done through Additive White Gaussian Noise (AWGN) channel. At the receiver side, FFT operation is performed on the received signal to convert time domain signal to frequency domain signal. Demodulation operation is performed using BPSK demodulator. Which get further processed by Random de-interleaver. Keeping coding techniques as RS code, interleaver as Random, Modulation technique is changed to QPSK and then to QAM. Then performance parameters such as BER (Bit Error Rate), power consumption are plotted. Now keeping the coding technique as RS encoder with BPSK/QPSK/QAM modulation technique, interleaving technique is changed to matrix. Performance parameters are again simulated. Now data is encoded with Turbo code, interleaving is done through random interleaver. Three different modulation techniques BPSK/QPSK/QAM are used as modulation techniques and then transmitted through AWGN channel. At the receiving end, three different modulation techniques BPSK/QPSK/QAM are used as demodulators. Then the output is de-interleaved through Random de-interleaver then decoded to get the original bit stream. Now keeping Turbo code as encoding technique to encode binary data stream, interleaver is 
changed to Matrix. Then demodulated through BPSK/QPSK/QAM and decoded to get original data. Performance parameters are simulated and compared as shown in table1.

\section{PERFormance ANALYSIS OF IDMA OFDM MIMO WITH VARIOUS CODING, INTERLEAVERS AND MODULATION TECHNIQUES - RESUlTS AND DISCUSSION}

The simulation of IDMA OFDM MIMO for various coding (RS code, Turbo code) with different modulation techniques (BPSK, QPSK, QAM) and different interleavers (Random, Matrix) are investigated for various parameters such as Bit Error Rate (BER) and power consumption. In UWSN nodes are battery operated so power consumption is considered as an important parameter.

A. RS Code with Random Interleaver and BPSK, QPSK, QAM modulations

In fig. 2 the power consumption of IDMA OFDM MIMO for underwater acoustic communication by taking RS code with Random interleaver and BPSK, QPSK and QAM modulation techniques are compared. The power consumption for BPSK and QPSK is approximately 35dB for $\mathrm{Eb} / \mathrm{No} 12$ to 14 and for QAM it is approximately $20 \mathrm{~dB}$ for $\mathrm{Eb} / \mathrm{No} 12$ to $14 \mathrm{~dB}$.

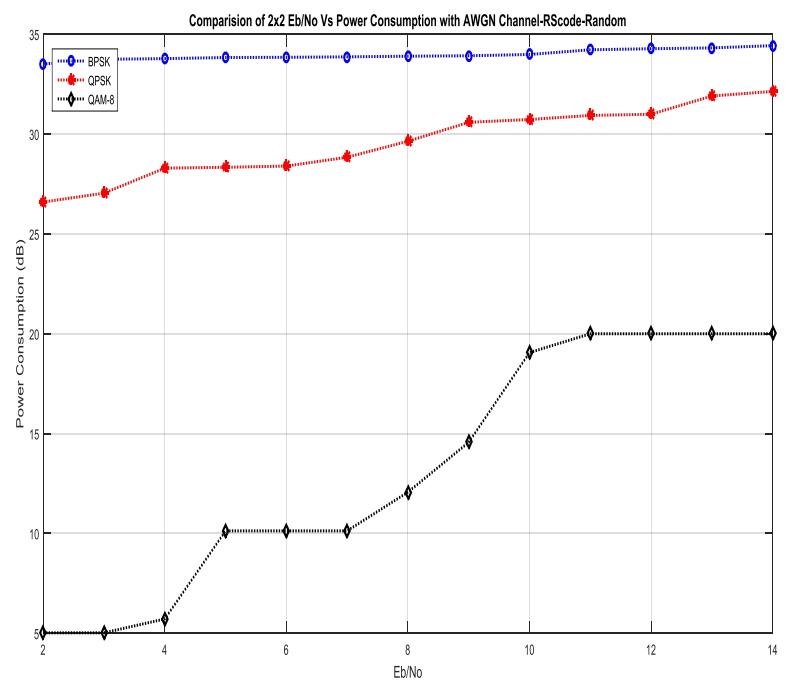

Fig.2. Power Consumption for RS code with Random Interleaver and BPSK, QPSK and QAM Modulation Technique

In fig. 3 the BER of IDMA OFDM MIMO for underwater acoustic communication by taking RS code with random interleaver and BPSK, QPSK and QAM modulation techniques are compared. BER for BPSK, QPSK and QAM is approximately $10^{-3}$ for SNR 12 to $14 \mathrm{~dB}$.

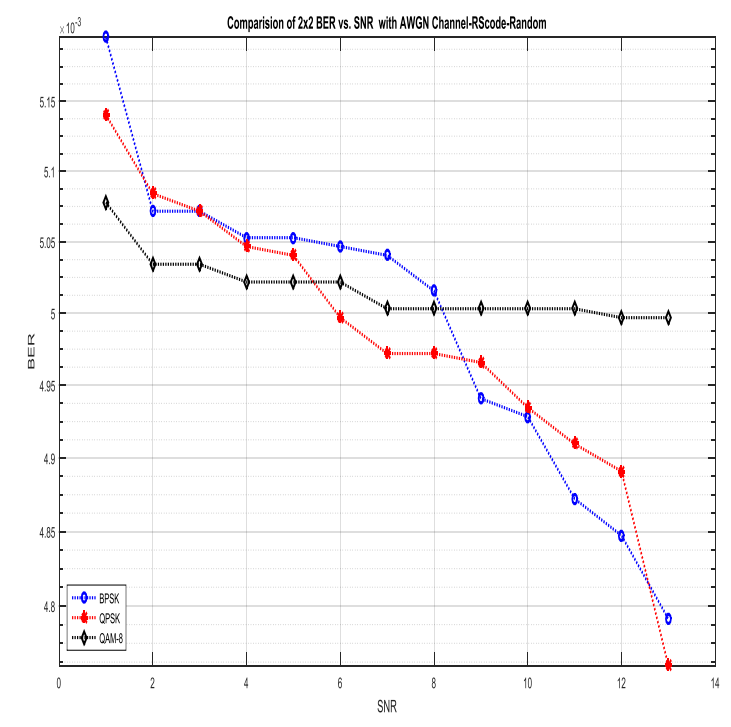

Fig.3. BER for RS code with Random Interleaver and BPSK, QPSK and QAM Modulation Technique QAM modulations

In fig.4 the power consumption of IDMA OFDM MIMO for underwater acoustic communication by taking RS code with matrix interleaver and BPSK, QPSK and QAM modulation techniques are compared. The power consumption for BPSK and QPSK is approximately $35 \mathrm{~dB}$ for $E_{b} / N_{o} 12$ to 14 and for QAM is approximately $20 \mathrm{~dB}$ for $\mathrm{E}_{\mathrm{b}} / \mathrm{N}_{\mathrm{o}} 12$ to $14 \mathrm{~dB}$.

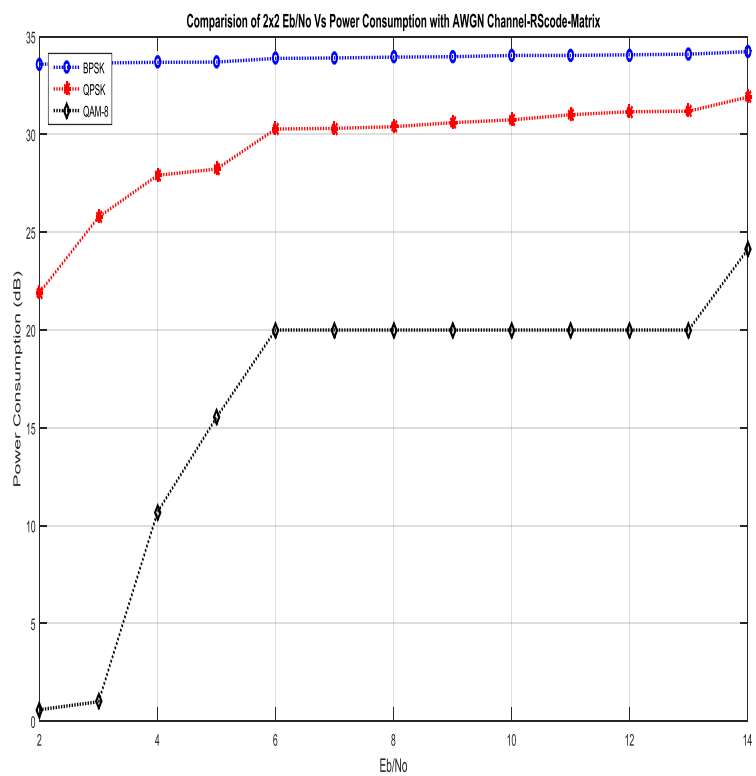

Fig.4. Power Consumption for RS code with Matrix Interleaver and BPSK, QPSK and QAM Modulation Technique

In fig.5 the BER of IDMA OFDM MIMO for underwater acoustic communication by taking RS code with matrix interleaver and BPSK, QPSK and QAM modulation techniques are compared. BER for BPSK, QPSK and QAM it is approximately $10^{-3}$ for $\mathrm{E}_{\mathrm{b}} / \mathrm{N}_{\mathrm{o}} 12$ to $14 \mathrm{~dB}$.
B. RS Code with Matrix Interleaver and BPSK, QPSK, 


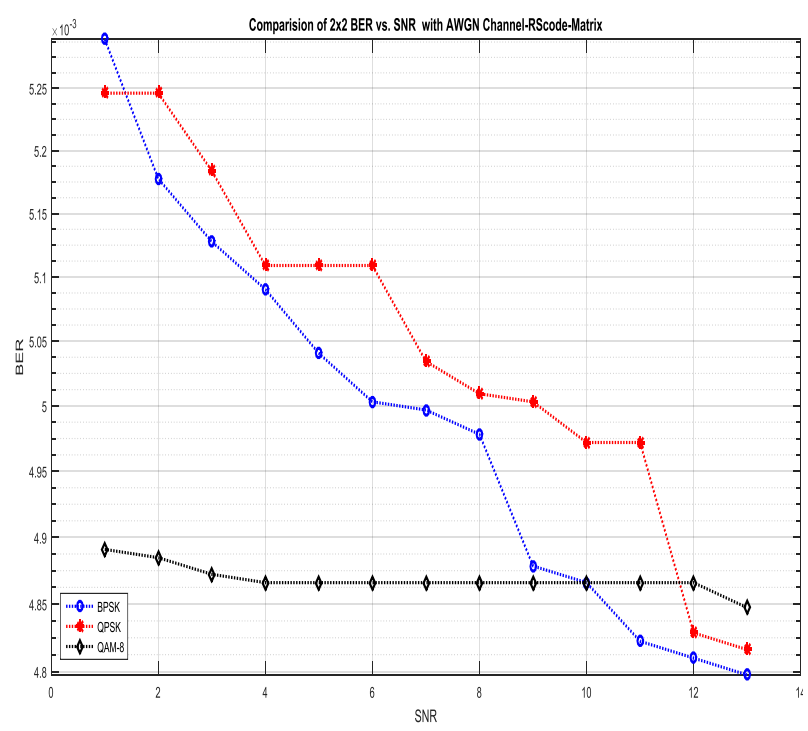

Fig.5. BER for RS code with Matrix Interleaver and BPSK, QPSK and QAM Modulation Technique

C. Turbo Code with Random Interleaver and BPSK, QPSK, QAM modulations

In fig. 6 the power consumption of IDMA OFDM MIMO for underwater acoustic communication by taking Turbo code with Random interleaver and BPSK, QPSK and QAM modulation techniques are compared. The power consumption for BPSK, QPSK and QAM is approximately 34 to $35 \mathrm{~dB}$ for $\mathrm{E}_{\mathrm{b}} / \mathrm{N}_{\mathrm{o}} 12$ to $14 \mathrm{~dB}$.

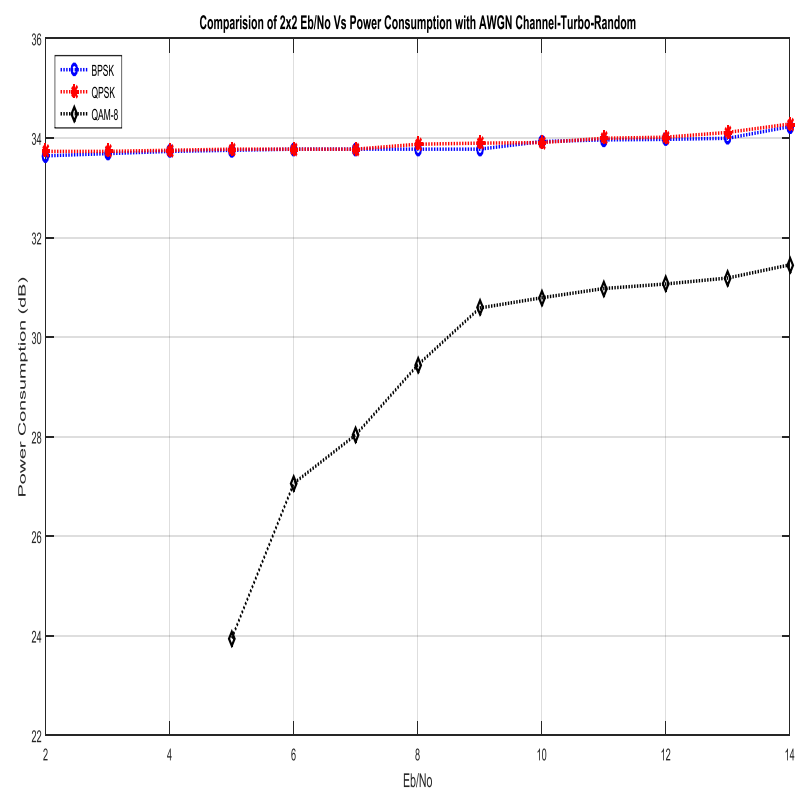

Fig.6. Power Consumption for Turbo code with Random Interleaver and BPSK, QPSK and QAM Modulation Technique

In fig.7 the BER of IDMA OFDM MIMO for underwater acoustic communication by taking Turbo code with Random interleaver and BPSK, QPSK and QAM modulation techniques are compared. BER for BPSK is $10^{-6}$ and for QPSK and QAM it is approximately $10^{-3}$ for $\mathrm{E}_{\mathrm{b}} / \mathrm{N}_{\mathrm{o}} 12$ to $14 \mathrm{~dB}$.

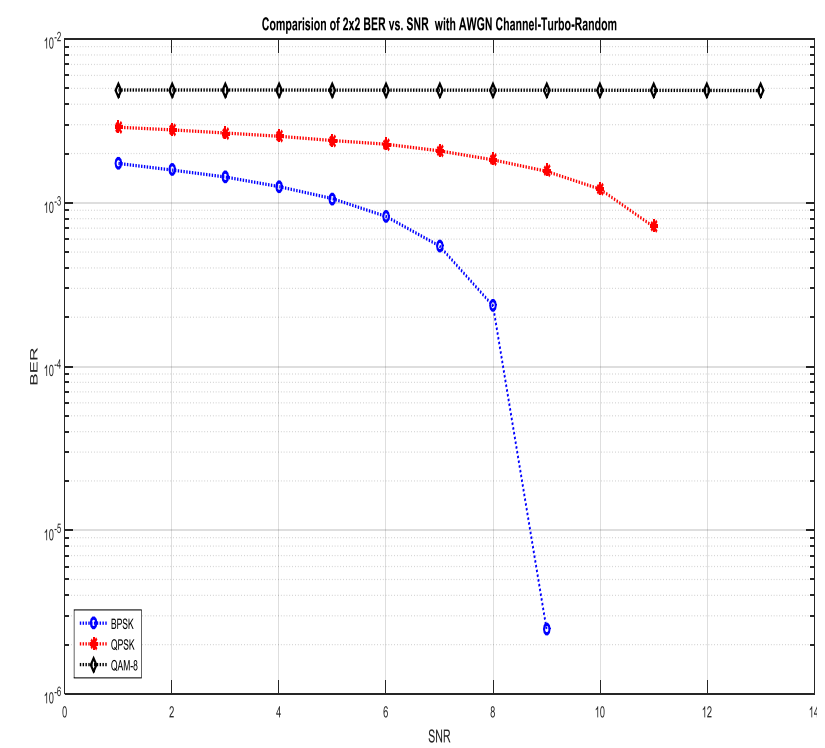

Fig.7. BER for Turbo code with Random Interleaver and BPSK, QPSK and QAM Modulation Technique

D. Turbo Code with Matrix Interleaver and BPSK, QPSK, QAM modulations

In fig.8 the power consumption of IDMA OFDM MIMO for underwater acoustic communication by taking Turbo code with Matrix interleaver and BPSK, QPSK and QAM modulation techniques are compared. The power consumption for BPSK, QPSK and QAM is approximately 34 to $35 \mathrm{~dB}$ for $\mathrm{E}_{\mathrm{b}} / \mathrm{N}_{\mathrm{o}} 12$ to $14 \mathrm{~dB}$.

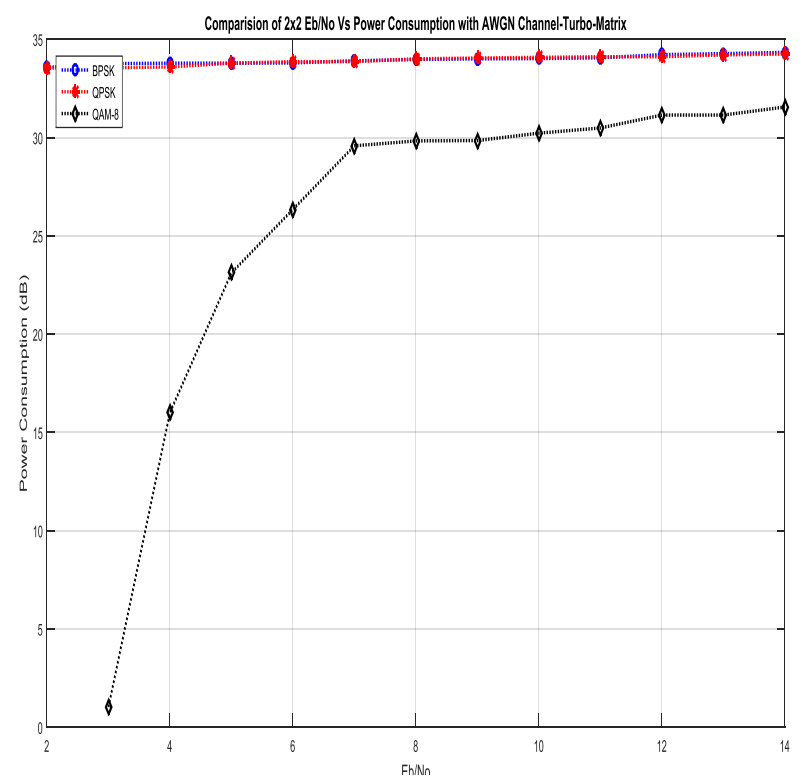

Fig.8. Power Consumption for Turbo code with Matrix Interleaver and BPSK, QPSK and QAM Modulation Technique

In fig.9 the BER of IDMA OFDM MIMO for underwater acoustic communication by taking Turbo code with Matrix interleaver and BPSK, QPSK and QAM modulation techniques are compared. BER for BPSK, QPSK and QAM is approximately $10^{-3}$ for $\mathrm{E}_{\mathrm{b}} / \mathrm{N}_{\mathrm{o}} 12$ to $14 \mathrm{~dB}$. 


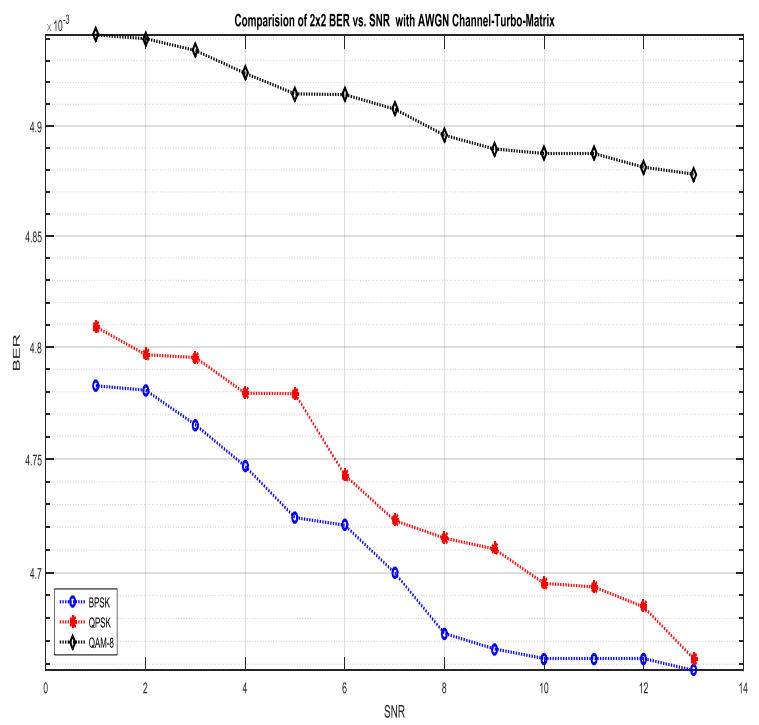

Fig.9. BER for Turbo code with Matrix Interleaver and BPSK, QPSK and QAM Modulation Technique

\section{COMPARISON OF PERFORMANCE PARAMETERS}

In this section Bit Error Rate and power consumption of IDMA OFDM MIMO using various coding techniques (RS code/Turbo code), Random/Matrix interleavers and different modulation techniques are compared.

Table 1. Comparison of Performance Parameters

\begin{tabular}{|c|c|c|c|c|}
\hline $\begin{array}{c}\text { Modulation } \\
\text { Technique }\end{array}$ & $\begin{array}{c}\text { Coding } \\
\text { Technique }\end{array}$ & Interleavers & BER & $\begin{array}{c}\text { Power } \\
\text { Consumption } \\
(\mathrm{dB})\end{array}$ \\
\hline BPSK & RS & Random & $10^{-3}$ & 35 \\
\hline QPSK & RS & Random & $10^{-3}$ & 34 \\
\hline QAM & RS & Random & $10^{-3}$ & $\mathbf{2 0}$ \\
\hline BPSK & RS & Matrix & $10^{-3}$ & 35 \\
\hline QPSK & RS & Matrix & $10^{-3}$ & 34 \\
\hline QAM & RS & Matrix & $10^{-3}$ & $\mathbf{2 0}$ \\
\hline BPSK & Turbo & Random & $\mathbf{1 0}^{-6}$ & 34 \\
\hline QPSK & Turbo & Random & $10^{-3}$ & 34 \\
\hline QAM & Turbo & Random & $10^{-3}$ & 32 \\
\hline BPSK & Turbo & Matrix & $10^{-3}$ & 35 \\
\hline QPSK & Turbo & Matrix & $10^{-3}$ & 35 \\
\hline QAM & Turbo & Matrix & $10^{-3}$ & 32 \\
\hline
\end{tabular}

\section{CONCLUSION}

The paper investigates the blend of IDMA OFDM MIMO for channel coding techniques with different interleavers and modulation techniques for reliable underwater acoustic communication. The simulation results reveal that the two combination QAM with RS code (Random interleaver, Matrix interleaver) have BER of $10^{-3}$ and power consumption of up to $20 \mathrm{~dB}$ at SNR 12$14 \mathrm{~dB}$. The combination of BPSK with Turbo code (Random interleaver) improves BER $10^{-6}$ however power consumption is increase up to $35 \mathrm{~dB}$ at the same SNR. For the energy efficient underwater communication power consumption play a key role. But simulation results give tradeoff between BER and power consumption.

\section{REFERENCES}

[1] G. Barreto, D. Simao, M. E. Pellenz, R. D. Souza, E.Jamhour, M. C. Penna, G. Brante, and B. S. Chang, "Energy-Efficient Channel Coding Strategy for Underwater Acoustic Networks", Sensors, vol. 17, pp.116,2017

[2] S. More and K. K. Naik, "Modelling and Simulation of IDMA-OFDM for Underwater Acoustic Communication", Defence Science Journal, vol. 65, pp.307-311, 2015.

[3] H. Luo, K. Wu; R. Ruby and L. M. Ni, "Simulation and Experimentation Platforms for Underwater Acoustic Sensor Networks: Advancements and Challenges", ACM Computing Surveys, vol.50, pp.28:1-28:44, 2017

[4] K. Pelekanakis and M. Chitre, "New sparse adaptive algorithms based on the natural gradient and the L0norm", IEEEJ. Ocean. Eng., vol. 38, pp.323-332, 2013.

[5] Y. V. Zakharov and A. K. Morozov, "OFDM transmission without guard interval in fast-varying underwater acoustic channels," IEEE J. Ocean. Eng., vol. 40, pp.144-158, 2015.

[6] Z. Wang, S. Zhou; J. Catipovic; and P. Willett, "Asynchronous multiuser reception for OFDM in underwater acoustic communications," IEEE Trans. Wireless Commun., vol.12, pp.1050-1061, 2013.

[7] S. S. Shahapur and R. Khanai "Underwater Sensor Network at physical, data link and network - layer a survey," $4^{\text {th }}$ IEEE international conference on communication and signal processing, April 2015

[8] Y. Chen; Z.-H. Wang; L. Wan; H. Zhou; S. Zhou; and X. $\mathrm{Xu}$, "OFDM modulated dynamic coded cooperation in underwater acoustic channels," IEEE J. Ocean. Eng., vol.40, pp. 159-168, 2015.

[9] Uddin, Md. A., Rashid, M., Rahman, Md. M.: 'Priority Metric based Ad Hoc Routing for Underwater Sensor Network', I. J. Computer Network and Information Security, 2013, 12, pp. 1-11, DOI: DOI: 10.5815/ijcnis.2013.12.01

[10] Z. Chen, J. Wang and Y. R. Zheng, "Frequency-Domain Turbo Equalization with Iterative Channel Estimation for MIMO Underwater Acoustic Communications", IEEE Journal of Ocean Eng., vol.42, pp.711-721, 2017.

\section{Authors' Profiles}

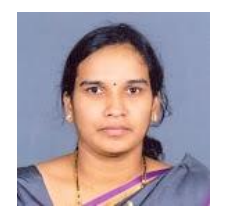

Rajashri Khanai received her $\mathrm{PhD}$ in Error Correction Coding and Cryptography for wireless communication from the Visvesvaraya Technological University, Belagavi, India. Her research interests include error control codes, cryptography, and machine learning applications to signal analysis. She is currently working as Professor in the Department of Electronics and Communication Engineering, KLE's Dr. M. S. Sheshgiri College of Engineering and Technology, Belagavi, Karnataka, India. She has published over 25 research papers. Dr. Rajashri is a member of IEEE.

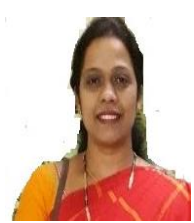

Salma S. Shahapur is currently pursuing her $\mathrm{PhD}$ from the Visvesvaraya Technological University, Belagavi, India in the field of optimal algorithms for various issues in the underwater communication. She received her master of engineering from Gogte Institute of 
technology Belagavi in Digital Communication and Networking. She has published 2 research papers on underwater communication in conferences. She is currently Assistant Professor in the Department of Electronics and Communication Engineering, Jain college of Engineering, Belagavi, Karnataka, India.

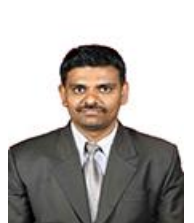

Dattaprasad Torse has received his $\mathrm{PhD}$ from the Visvesvaraya Technological University, Belagavi, India in the field of biomedical (EEG) signal analysis for seizure detection/classification applications. $\mathrm{He}$ received his master of engineering from Amravati University in Digital Electronics. He has published over 15 research papers on EEG signal analysis in journal and conferences. $\mathrm{He}$ is currently Assistant Professor in the Department of Electronics and Communication Engineering, KLS Gogte Institute of Technology, Belagavi, Karnataka, India. Dr. Torse is a member of IEEE.

How to cite this paper: Rajashri Khanai, Salma S. Shahapur, Dattaprasad Torse,"Performance Analysis of Underwater Acoustic Communication using IDMA-OFDM-MIMO with Reed Solomon and Turbo Code", International Journal of Computer Network and Information Security(IJCNIS), Vol.10, No.12, pp.41-46, 2018.DOI: 10.5815/ijcnis.2018.12.05 